\title{
Construction and Comprehensive Analysis of the Competing Endogenous Rna Network in Endometrial Adenocarcinoma
}

\section{Chong Feng}

Shengjing Hospital of China Medical University

Lei Cui ( $\square$ leicui_cmu@126.com)

Department of medical information, China medical university https://orcid.org/0000-0002-8671-3444

Zhen Jin

Shengjing Hospital of China Medical University

Lei Sun

Shengjing Hospital of China Medical University

Xiaoyan Wang

Shengjing Hospital of China Medical University

Xinshu Chi

Shengjing Hospital of China Medical University

Qian Sun

Shengjing Hospital of China Medical University

Siyu Lian

Shengjing Hospital of China Medical University

\section{Research}

Keywords: endometrial carcinoma, competing endogenous RNAs co-expression, weighted gene coexpression network analysis, hub gene

Posted Date: August 31st, 2020

DOI: https://doi.org/10.21203/rs.3.rs-61610/v1

License: (c) (i) This work is licensed under a Creative Commons Attribution 4.0 International License.

Read Full License

Version of Record: A version of this preprint was published at BMC Genomic Data on February 6th, 2022. See the published version at https://doi.org/10.1186/s12863-022-01028-y. 


\section{Abstract}

Background: Endometrial carcinoma (EC) is one of the most common gynecological malignant tumors. In this study, we constructed gene co-expression networks to identify key modules and hub genes involved in the pathogenesis of EC.

Methods: The RNA profiles of adenocarcinoma and non-adenocarcinoma tissues of patients with EC from The Cancer Genome Atlas were analyzed. MicroRNA, mRNA, and long non-coding RNA networks were constructed based on the differentially expressed RNAs between the two tissues. RNAs from the competing endogenous RNA (ceRNA) networks were then used to construct co-expression networks by weighted gene co-expression network analysis. Gene Ontology (GO) and Kyoto Encyclopedia of Genes and Genomes enrichment analyses were performed by the Database for Annotation, Visualization and Integrated Discovery tool.

Results: The MEturquoise module was found to be significantly related to hypertension and the MEbrown module was significantly related to the history of other malignancies. Functional enrichment analysis showed that the MEturquoise module was associated with the $\mathrm{GO}$ biological process terms of transcription from RNA polymerase II promoter, positive regulation of male gonad development, endocardial cushion development, and endothelial cell differentiation. The MEbrown module was associated with GO terms DNA binding, epithelial-to-mesenchymal transition, and transcription from RNA polymerase II promoter. A total of 10 hub genes were identified and validated at transcriptional and translational levels.

Conclusions: The identified ceRNAs may play a critical role in the progression and metastasis of EC and are thus candidate therapeutic targets and potential prognostic biomarkers. The two modules constructed further provide a useful reference that will advance understanding of the mechanisms of tumorigenesis in EC.

\section{Introduction}

Endometrial carcinoma (EC) is one of the most common gynecological tumors worldwide, and its incidence has been on the rise every year in both developed and developing countries [1]. Kessler et al. [2] reported that the incidence of EC remained unchanged in women aged 50-74 years from 1992 to 2002, but increased by $2.5 \%$ annually from 2006 to 2012 . This significant increase in the incidence of EC in recent years is attributed to a change of lifestyles and dietary structure, along with informal hormone replacement therapy and abuse of sex hormones; however, the use of vaginal estrogen was found to not increase the incidence of EC [5]. Moreover, patients diagnosed with EC are becoming younger, representing a serious threat to women's health $[3,4]$. In 2014, The World Health Organization classified EC according to histological type as endometrioid, serous, mucinous, clear cell, neuroendocrine, mixed, undifferentiated/dedifferentiated, and others. Among these types, adenocarcinoma, clear cell carcinoma, serous papillary adenocarcinoma, squamous cell carcinoma, and undifferentiated carcinoma are highly 
malignant, whereas squamous cell carcinoma and undifferentiated carcinoma are relatively rare. EC has a known hereditary component. In patients without metastatic tumors, the 5-year survival rates range from $74-91 \%$. However, there is a lack of statistical data based on appropriate methods for large-scale screening of EC in an asymptomatic population, and there is no relevant guideline for screening of EC in the asymptomatic general population or medium-risk population. In contrast to other gynecological tumors such as ovarian cancer and cervical cancer that are associated with highly specific landmark molecules such as CA125, HE4, and SCC [11-13], no such markers are available for EC, which is a challenge for clinical diagnosis. The diagnosis of EC thus mainly depends on imaging, immunohistochemistry, pathology, and hysteroscopy $[6,14-16]$. At present, it is considered that transvaginal ultrasound measurement of endometrial thickness [6-8] and endometrial aspiration cytological examination are feasible technologies for EC screening. Dilatation and curettage $[9,10]$ and hysteroscopy are also potentially effective screening methods, but are highly invasive and therefore not recommended. Therefore, further effort is needed to identify candidate molecular markers to improve the screening and early diagnosis of EC, as well as to provide insight into the pathogenic mechanism.

Salmena et al. [17] proposed the competing endogenous RNA (ceRNA) hypothesis in 2011, suggesting that mRNAs, transcribed pseudogenes, and long non-coding RNAs (IncRNAs) form a ceRNA network through microRNA (miRNA) response elements (MREs), which plays an important role in tumor formation. The traditional view of gene regulation is that an miRNA binds to its target mRNA to affect the translation and stability of the target gene. This binding of the miRNA at the MRE can induce the target gene mRNA to degrade or inhibit its translation into a protein to regulate gene expression at the posttranscriptional level $[18,19]$. Seitz et al. [20] confirmed that transcribed pseudogenes and IncRNAs could be competitively bound to miRNA at the binding sites, resulting in down-regulation of the activity and quantity of the miRNA, which would remove the inhibitory effect on the downstream target mRNA. Based on this background, we aimed to identify potential markers of EC by identifying differentially expressed RNAs in endometrial adenocarcinoma and non-cancer tissues, which were used to construct a ceRNA network. For this purpose, we used available gene expression data of EC patients from The Cancer Genome Atlas (TCGA), a joint project supervised by the National Cancer Institute and the National Human Genome Research Institute, which aims to use high-throughput genomic analysis techniques to facilitate research toward gaining a better understanding of cancer, and ultimately improve the ability to prevent, diagnose, and treat cancer. Based on the constructed network, we conducted weighted gene coexpression network analysis (WGCNA), which was combined with clinical feature screening modules, and functional annotation of relevant genes was conducted with enrichment of Gene Ontology (GO) terms and Kyoto Encyclopedia of Genes and Genomes (KEGG) pathways. These results can serve as a useful reference for screening adenocarcinoma with endometrium-specific RNAs representing ceRNAs that can regulate target genes, which can facilitate further research into the pathogenesis, clinical diagnosis, and accurate targeted therapy of EC.

\section{Methods}

\section{Data collection}


The transcripts of the TCGA-UCEC (disease type: endometrial adenocarcinoma) database were downloaded, comprising count and miRNA-Seq data expressed by RNAs. The downloaded data were preprocessed to form a gene expression matrix, the mRNAs and IncRNAs in the count data matrix were separated, and the miRNA matrix was added to obtain three separate matrices for co-expression analyses.

\section{Differential gene expression analysis}

We used R 3.5.1 software with the R packages edger, gplots, and pheatmap to extract differentially expressed genes (DEGs) from the dataset and draw volcanic and thermal maps. The criteria for DEG identification were an absolute log fold-change value $>2$ and false discovery rate (adjusted $p$-value) < 0.01. The differentially expressed IncRNAs and miRNAs were then compared in the miRcode database to screen out the IncRNA-miRNA relationships specific to endometrial adenocarcinoma. After the compared miRNAs were modified for $3 p, 5 p$ standardization in the starBase database, their target genes were predicted using the miRDB, miRTarBase, and TargetScan tools, and then the miRNA-mRNA relationships of endometrial adenocarcinoma were obtained. The predicted target genes were intersected with the screened differentially expressed RNAs, and a Venn diagram was obtained using the venndiagram R package. After comparison with mRNAs, the miRNAs were compared with the selected differentially expressed IncRNAs to identify related IncRNA-miRNA pairs. Finally, the ceRNA network diagram of differentially expressed RNAs in EC was constructed with Cytoscape.

\section{WGCNA}

WGCNA was performed based on the constructed ceRNA network and patient characteristics, including hypertension, diabetes, history of colorectal cancer, history of other malignancies, and new tumor events. Randomly selected cancer samples from the TCGA database were clustered and the abnormal samples were removed according to a certain height. After loading the trait data, the removed samples were regrouped and linked to the trait data to make a heat map. A relationship matrix of the expression data was constructed, followed by a topology matrix according to the fitting indices ( $\mathrm{R}^{2}$ and mean connectivity with a soft threshold (power) value), and the final scale-free network. According to tom-based dissimilarity, the average method was used to cluster the genes, and the dynamic shearing tree method was used to identify the modules.

\section{GO and KEGG enrichment analysis}

The Database for Annotation, Visualization and Integrated Discovery (DAVID) v. 6.8 tool comprises a full Knowledgebase update to the sixth version of the original web-accessible programs. DAVID now provides a comprehensive set of functional annotation tools to interpret the biological meaning behind large lists of genes (https://david.ncifcrf.gov/home.jsp). KOBAS 3.0 is a web server for gene/protein functional annotation (Annotate module) and functional gene set enrichment (http://kobas.cbi.pku.edu.cn/). Genes from two of the identified modules showing significant associations with patient characteristics, MEbrown and Meturquiose, were respectively input in the DAVID database for functional annotation and 
enrichment from the GO biological process, cellular component, and molecular function terms, followed by KEGG pathway enrichment.

\section{Selection and verification of hub genes}

Highly connected nodes in a network are considered to play an important role in the stability of the network. Therefore, in the MEbrown and MEturquiose modules, the genes with nodal degree $>3$ were selected as candidate hub genes. These hub genes were analyzed with respect to their relationship to patient survival in GEPIA (http://gepia.cancer-pku.cn/), a newly developed interactive web server for analyzing RNA-seq expression data of 9,736 tumors and 8,587 normal samples from the TCGA and GTEx projects using a standard processing pipeline. In this study, genes with a log rank value $<0.05$ were selected from the two modules as potential hub genes in the endometrial adenocarcinoma genetic network. The transcriptional levels of the hub genes were verified in The Human Protein Atlas database (https://www.proteinatlas.org/), a Swedish-based program initiated in 2003 with the aim to map all of the human proteins in cells, tissues, and organs using integration of various omics technologies, including antibody-based imaging, mass spectrometry-based proteomics, transcriptomics, and systems biology.

\section{Genetic alteration of hub genes}

The genomic changes in candidate hub genes and their correlations were assessed with cBioPortal for Cancer Genomics, which provides tools for the visualization, analysis, and download of large-scale cancer genomics datasets. Complex cancer genomic profiles can be easily obtained using the query interface of the portal, enabling researchers to explore and compare genetic alterations across samples, and to search for potential targets of anti-tumor drugs.

\section{Results}

\section{Data download}

RNA-seq data of endometrial adenocarcinoma were downloaded from the TCGA dataset, comprising a total of 425 samples, including 19 normal samples and 406 cancer samples. The miRNA-seq data comprised a total of 423 samples, including 18 normal samples and 405 cancer samples.

\section{DEG identification}

A total of 2,731 differentially expressed mRNAs were obtained, 1,566 of which were up-regulated and 1,165 were down-regulated. A total of 979 differentially expressed IncRNAs were obtained, 611 of which were up-regulated and 368 were down-regulated. A total of 170 differentially expressed miRNAs were obtained, 127 of which were up-regulated and 43 were down-regulated (Fig. 1, Supplementary Material 19). The predicted target genes were intersected with the screened differentially expressed RNAs, and visualized with a Venn diagram. The constructed ceRNA network diagram of differentiated RNAs in endometrial adenocarcinoma is shown in Fig. 2, including 90 mRNAs, 81 IncRNAs, and 25 miRNAs. 


\section{WGCNA}

Twenty-seven cancer samples were randomly selected from the TCGA data for further WGCNA analysis of the 196 differentially expressed RNAs in the ceRNA network. The heat map of the correlations between patient characteristics and RNA expression is shown in Fig. 3a. A total of seven modules were constructed: MEbrown, MEblack, MEyellow, MEgreen, MEred, MEblue, and MEturquiose (Fig. 3b). Based on the final scale-free network constructed (Fig. 3c-d), the GeneSignificance( GS) and Module membership(MM) values of the modules were calculated according to combined analysis of modules and trait data along with mining the modules and key genes that play a key role in endometrial adenocarcinoma (Supplementary Material 10). The modules and trait data were found to be significantly correlated, and the heat map of these relationships is shown in Fig. 4a. Overall, this analysis indicated that hypertension was positively correlated with the MEturquiose module $(p=0.005)$, and history of other malignancy was positively correlated with the MEbrown module $(p=0.02)$. The gene clustering tree and heat map of the network are shown in Fig. $4 \mathrm{~b}$, and the genes in these two modules were input into Cytoscape (3.7.0) to obtain the weighted ceRNA network graph (Fig. 5).

\section{GO and KEGG enrichment analysis}

The GO terms related to these topology modules indicated important functional features. The MEbrown topology module was significantly enriched in four GO terms (Supplementary Material 11): GO 0043565 $(p=2.44 \mathrm{E}-04)$, closely related to the molecular function of sequence-specific DNA binding; G0 0080301 $(p=0.012)$, closely related to the molecular function of DNA binding/bending; GO $001837(p=0.024)$, associated with the biological process of epithelial-to-mesenchymal transition; and G0 $0045944(p=$ 0.029), associated with the biological process of the positive regulation of transcription from RNA polymerase II promoter (Fig. 6b). The MEturquiose module was significantly enriched in $12 \mathrm{GO}$ terms (Supplementary Material 12). A circle diagram was used to represent the top five GO enrichment terms with the most significant differences, which were all associated with biological process terms (Fig. 6a): GO 0045944 ( $p=0.006)$, associated with positive regulation of transcription from RNA polymerase II promoter; GO 2000020 ( $p=0.009$ ), associated with positive regulation of male gonad development; GO 0000122 ( $p=0.011)$, associated with negative regulation of transcription from RNA polymerase II promoter; GO $0003197(p=0.012)$, associated with endocardial cushion development; and G0 0045446 $(p=0.014)$, associated with endothelial cell differentiation (Fig. 7). Pathway enrichment analysis (KOBAS 3.0) of the genes in the MEbrown module showed that RECK and E2F1 were enriched in the miRNA in cancer pathway $(p<0.05)$, with no significant enrichment detected for the other genes. However, there was no significant pathway enrichment for genes in the MEturquiose module (Supplementary Material 13).

\section{Selection and verification of hub genes}

A total of 33 and nine genes in the MEbrown and MEturquiose module, respectively, showed a nodal degree $>3$ and were thus selected as candidate hub genes. GEPIA analysis of these genes (log rank < 0.05 ) further identified C10orf91, LINC00303, DIRC3, DLG3-AS1, ARHGEF38-IT1, and CCNE1 as hub genes 
in MEturquoise, and RECK, MDGA1, CFL2, TGFBR3, and TPM2 as hub genes in MEBrown (Fig. 8). The transcriptional levels of these candidate hub genes were verified in The Human Protein Atlas database. There was no significant difference in the protein expression of MDGA1 between normal tissues and endometrial adenocarcinoma tumor tissues. Unfortunately, C10orf91, LinC00303, and DIRC3 are IncRNAs, DLG3-AS1 is an antisense gene, and ARHGEF38-IT1 is a sense-intronic InCRNA; therefore, there are no data related to their expression in the Human Protein Atlas (Fig. 9).

\section{Genetic alteration of hub genes}

After MDGA1 was removed, the remaining candidate hub genes (RECK, CFL2, TGFBR3, TPM2, and C10ORF91) affecting the survival rate of endometrial adenocarcinoma in the MEbrown module and MEturquoise module were selected. Based on the oncoPrint map in cBioportal, these 10 hub genes were found to be altered in $73(31 \%)$ of 232 queried patient samples (Fig. 10b), with the greatest degree of alterations detected for CCNE1, RECK, CFL2, TGFBR3, TPM2, and LINCO0303, detected in $11 \%, 7 \%, 6 \%$, $7 \%, 6 \%$, and $6 \%$ of the samples, respectively. The main types of alterations detected were missense mutation, amplification, and mRNA upregulation, although only amplification was found for LINC00303 alterations (Fig. 10a). Fig. 10c demonstrates the relationship of the 10 genes and the other 50 most frequently altered neighboring genes; however, only CFL2, CCNE1, and TPM2 had connections with these 50 genes. There was no known drug targeting these 10 hub genes, indicating promising targets of new cancer drugs. In the MEturquiose module, C10orf91, LINC00303, DIRC3, DLG3-AS1, and ARHGEF38-IT1 are all IncRNAs with no protein expression data available. The correlation analysis of the mRNAs RECK, CFL2, TGFBR3, TPM2, and CCNE1 in the GEPIA website demonstrated a significant negative correlation between $C C N E 1$ and the other hub genes in the MEbrown module; the other hub genes in the MEbrown module were all significantly positively correlated with each other (Fig. 11).

\section{Discussion}

EC is a common malignant tumor in women, and is one of the three major malignancies of the female reproductive system that seriously affects the quality of life and health of women [21]. Although the prognosis of low-grade EC (grades 1 and 2) is better than that of high-grade EC (grade 3) [22], some patients with low-grade EC still have recurrence and a poor prognosis. Lu et al. [23] recently reported that the incidence of EC was associated with folate-mediated one-carbon metabolism according to a Cox proportional hazards model: with the increase of total folate, natural folate, B6, and B12 intake, there was a significantly increased risk of EC. EC risk has also been associated with greater lifetime number of years of menstruation [24]. Indeed, EC risk is closely related to age, and $90 \%$ of cases occur in women older than 45 years $[25,26]$. In addition, the risk of EC in patients with diabetes is three times higher than that of the general population, and women with metabolic diseases such as hyperglycemia and obesity should be screened regularly [27, 28]; indeed, obesity, diabetes, and hypertension are together referred to as the "triad of endometrial cancer" [29-31]. Consistently, in the present study, we found a positive correlation between hypertension and the MEturquiose module in the WGCNA. Moreover, GO enrichment 
analysis showed significant enrichment of G0:0003197, linked to the biological processes of endocardial cushion development.

Soisson et al. [32] conducted a retrospective cohort study, and found that heart disease and other circulatory system conditions were the main causes of death of patients with $\mathrm{EC}$, and patients receiving radiation therapy and/or chemotherapy were found to have a higher risk of heart disease. The results of the present study can provide insight into this apparent relationship between EC and cardiovascular disease from the genetic level. Most IncRNAs are transcribed by RNA polymerase II, which exists in the cytoplasm of eukaryotes, into mRNA primary transcripts. All of the genes in the MEbrown module except for CCNE1 were IncRNAs, which also explains the significant correlation for G0:0045944 linked to positive and negative regulation of transcription from RNA polymerase II promoter, respectively.

Although there has been no large population-based case-control study on the correlation between EC and other tumors, Schildkraut et al. [33] found a significant association between breast cancer and ovarian epithelial tumor with EC in familial cases. In addition, Liu et al. [34] found that overexpression of syncytin1 promoted the invasion and metastasis of EC cells by activating epithelial-to-mesenchymal transitionrelated pathways. Consistently, in this study, the WGCNA confirmed that a history of other malignancies was positively correlated with the MEbrown module. Further, GO analysis of the MEbrown module showed significant enrichment of G0:001837, related to epithelial-to-mesenchymal transition, a wellknown important process of tumor metastasis. Moreover, the KEGG analysis showed that RECK and E2FI in the MEbrown module are enriched in the pathways related to miRNA in cancer. RECK is regulated by miR-21 and is involved in the invasion and metastasis of glioblastoma, and has also been shown to affect the survival rate of patients with this type of tumor. E2F1 is regulated by miR-20a, miR-106b, and miR-330 in prostate cancer and affects cell epigenetic changes related to cell growth (resistance to apoptosis). There is evidence that exocrine bodies are involved in tumor growth in prostate cancer, glioblastoma, and breast cancer [35-37]. During the process of tumor metastasis, the tumor tissue can secrete exosomes to the metastatic part in advance to make the microenvironment suitable for tumor growth [38]. The discovery of the MEbrown module provides a molecular basis for studying the relationship between EC and tumorigenesis processes of other tumor types at the genetic level. Based on our results, we speculate that factors such as RECK and E2F1 in the MEbrown module directly affect the invasion and metastasis of EC through gene regulation, resulting in the occurrence of other malignant tumors, activation of proto-oncogenes in other parts through exosomes, or promotion of the metastasis of EC to other tissues through the tumor microenvironment.

Current treatment for EC largely depends on chemotherapy regimens and hormone-based therapy in combination with surgery and radiotherapy [39]. Traditional chemotherapy drugs include platinum-based anti-neoplastics, taxanes, nucleoside analogues, immune modulators, fibroblast growth factor receptor and tyrosine kinase inhibitors, small-molecule mTOR inhibitors, and drugs that trigger cell cycle arrest in the $\mathrm{G} 1$ phase [40-42]. With the discovery of biomarkers of EC, some targeted therapies such as bevacizumab have shown remarkable anticancer effects [43]. By screening the MEturquoise and MEbrown modules, we identified RECK, CFL2, TGFBR3, TPM2, C100RF91, LINC00303, DIRC3, DLG3-AS1, 
ARHGEF38-IT1, and CCNE1 as hub genes. The discovery of these two modules provides a basis for further research on the mechanism of the occurrence and development of EC. Moreover, the newly identified 10 hub genes might serve as prognostic biomarkers and therapeutic targets in the future. In addition, no known anti-tumor targets of the 10 hub genes were predicted in cBioportal, which could provide new ideas for the development of targeted drugs in EC.

Zhou et al. [44] experimentally confirmed that large intergenic non-coding ribonucleic acid-RoR is a ceRNA and acts as an miR-145 "sponge" to inhibit the mediation of the differentiation of stem cells by miR- 145 . Vallone et al. [45] reviewed the relationship between non-coding RNAs and oncogenesis (lung cancer, bladder cancer, kidney cancer, and melanoma) based on three years of PubMed records. To date, very few ceRNA network studies have been performed with regards to EC. In the present study, correlation analysis showed that CCNE1 in the MEturquiose module was significantly negatively correlated with all of the hub genes in the MEbrown module, while all of the hub genes in the MEbrown module were significantly positively correlated, indicating that the two modules are not only modules with respect to topology but also with respect to function, and the existence of two modules is reasonable. In the ceRNA expression network of DEGs, we also found that both CCNE1 and TPM2 are only directly regulated by mir-424, and are negatively regulated by each other in mir-424-centered ceRNA; however, they belong to two modules, indicating that the two modules do not exist in isolation, but rather have a certain genetic correlation. CFL2 is only directly regulated by mir-106a in the ceRNA expression network of DEGs. Linear regulation of TPM2 and CFL2 may be directly mediated by mir-106a/oxct1-as $1 /$ mir-424 or through the complex ceRNA network between mir-106a and mir-424. This finding provides a new strategy for investigating the mechanism of EC.

\section{Conclusions}

We analyzed the differentially expressed RNAs of endometrial adenocarcinoma based on TCGA data, and established a ceRNA network. The identified ceRNAs may play a critical role in the progression and metastasis of EC, and are thus candidate therapeutic targets and potential prognostic biomarkers. The two modules identified provided a valuable reference that will advance studies into understanding the mechanisms of tumorigenesis in EC.

\section{List Of Abbreviations}

EC endometrial carcinoma

TCGA The Cancer Genome Atlas

MiRNA microRNA

IncRNA long non-coding RNA

ceRNA competing endogenous RNA 
WGCNA weighted gene co-expression network analysis

GO Gene Ontology

KEGG Kyoto Encyclopedia of Genes and Genomes

DAVID Database for Annotation, Visualization and Integrated Discovery

MRE microRNA response element

DEG differentially expressed gene

\section{Declarations}

\section{Ethics approval and consent to participate}

Not applicable

\section{Consent for publication}

Not applicable

\section{Availability of data and material}

The datasets analyzed during the current study are available in the TCGA repository (Data generated in the study can be accessed from theadditional files)

\section{Competing interests}

The authors declare that they have no competing interests.

\section{Funding}

This study was not supported by any funding.

\section{Authors' contributions}

CF analyzed the patient data and have drafted the work, LC designed the work and substantively revised it ,ZJ interpretated the data .LS and XW approved the submitted version,XC and QS made substantial contributions to the conception,SL drawed the figures in the manuscript.All authors read and approved the final manuscript.

\section{Acknowledgements}

Not applicable 
None declared:This manuscript has not been published or presented elsewhere in part or in entirety and is not under consideration by another journal. We have read and understood your journal's policies, and we believe that neither the manuscript nor the study violates any of these. There are no conflicts of interest to declare.

\section{References}

1. Lortet-Tieulent J, Ferlay J, Bray F, Jemal A. International Patterns and Trends in Endometrial Cancer Incidence, 1978-2013. Jnci-Journal of the National Cancer Institute. 2018;110:354-61.

2. Constantine GD, Kessler G, Graham S, Goldstein SR. Increased Incidence of Endometrial Cancer Following the Women's Health Initiative: An Assessment of Risk Factors. Journal of Womens Health.

3. Sponholtz TR, Palmer JR, Rosenberg LA, Hatch EE, Adams-Campbell LL, Wise LA. Exogenous Hormone Use and Endometrial Cancer in US Black Women. Cancer Epidemiology Biomarkers Prevention. 2018;27:558-65.

4. Simin J, Tamimi R, Lagergren J, Adami HO, Brusselaers N. Menopausal hormone therapy and cancer risk: An overestimated risk? Eur J Cancer. 2017;84:60-8.

5. Crandall CJ, Hovey KM, Andrews CA, Chlebowski RT, Stefanick ML, Lane DS, Shifren J, Chen C, Kaunitz AM, Cauley JA, Manson JE. Breast cancer, endometrial cancer, and cardiovascular events in participants who used vaginal estrogen in the Women's Health Initiative Observational Study. Menopause-the Journal of the North American Menopause Society. 2018;25:11-20.

6. ACOG Committee. Opinion No. 734 Summary: The Role of Transvaginal Ultrasonography in Evaluating the Endometrium of Women With Postmenopausal Bleeding. Obstetrics gynecology. 2018;131:945-6.

7. Su QC, Sun Z, Lv GR. Contrast enhanced ultrasound in diagnosis of endometrial carcinoma and endometrial hyperplasia. Cell Mol Biol (Noisy-le-grand). 2018;64:88-91.

8. Kanmaz AG, Inan AH, Beyan E, Budak A: Importance of transvaginal ultrasonography before endometrial sampling in asymptomatic postmenopausal patients. Journal of gynecology obstetrics and human reproduction 2018.

9. Wu HHJ, Harshbarger KE, Berner HW, Elsheikh TM. Endometrial brush biopsy (Tao brush) - Histologic diagnosis of 200 cases with complementary cytology: An accurate sampling technique for the detection of endometrial abnormalities. Am J Clin Pathol. 2000;114:412-8.

10. Wu HHJ, Casto BD, Elsheikh TM. Endometrial brush biopsy - An accurate outpatient method of detecting endometrial malignancy. J Reprod Med. 2003;48:41-5.

11. Elias KM, Guo J, Bast RC. Jr.: Early Detection of Ovarian Cancer. Hematol Oncol Clin N Am. 2018;32:903-14.

12. Huang J, Chen J, Huang Q. Diagnostic value of HE4 in ovarian cancer: A meta-analysis. Eur J Obstet Gynecol Reprod Biol. 2018;231:35-42. 
13. Valenti G, Vitale SG, Tropea A, Biondi A, Lagana AS. Tumor markers of uterine cervical cancer: a new scenario to guide surgical practice? Updates in surgery. 2017;69:441-9.

14. Kobel M, Ronnett BM, Singh N, Soslow RA, Gilks CB, McCluggage WG. Interpretation of P53 Immunohistochemistry in Endometrial Carcinomas: Toward Increased Reproducibility. International journal of gynecological pathology: official journal of the International Society of Gynecological Pathologists. 2019;38(Suppl 1):123-31.

15. Xu GC, Wang DY, Ling XT, Lu HW, Lin RC, Shi YY, Zhang BZ. Diagnostic Value of Assessment of Cervical Involvement in Early-Stage Endometrial Adenocarcinoma: Comparison of Magnetic Resonance Imaging (MRI) Versus Hysteroscopy. Med Sci Monit. 2018;24:7952-7.

16. Murali R, Davidson B, Fadare O, Carlson JA, Crum CP, Gilks CB, Irving JA, Malpica A, Matias-Guiu X, McCluggage WG, et al. High-grade Endometrial Carcinomas: Morphologic and Immunohistochemical Features, Diagnostic Challenges and Recommendations. International journal of gynecological pathology: official journal of the International Society of Gynecological Pathologists. 2019;38(Suppl 1):40-63.

17. Salmena L, Poliseno L, Tay Y, Kats L, Pandolfi PP. A ceRNA Hypothesis: The Rosetta Stone of a Hidden RNA Language? Cel/ 2011, 146:353-358.

18. Sinha P, Jaiswal P, Jainarayanan AK, Brahmachari SK. Intronic miRNA mediated gene expression regulation controls protein crowding inside the cell. Gene. 2018;679:172-8.

19. Ergun S, Oztuzcu S. Oncocers: ceRNA-mediated cross-talk by sponging miRNAs in oncogenic pathways. Tumor Biology. 2015;36:3129-36.

20. Seitz H. Redefining MicroRNA Targets. Curr Biol. 2009;19:870-3.

21. Denschlag D, Ulrich UA. Uterine Carcinosarcomas - Diagnosis and Management. Oncology Research Treatment. 2018;41:675-9.

22. Alkushi A, Gilks CB. Description of a novel system for grading of endometrial carcinoma and comparison with existing grading systems - Reply. Am J Surg Pathol. 2005;29:1116-7.

23. Lu J, Trabert B, Liao LM, Pfeiffer RM, Michels KA: Dietary intake of nutrients involved in folatemediated one-carbon metabolism and risk for endometrial cancer. International journal of epidemiology 2018.

24. Gavrilyuk O, Braaten T, Weiderpass E, Licaj I, Lund E. Lifetime number of years of menstruation as a risk index for postmenopausal endometrial cancer in the Norwegian Women and Cancer Study. Acta Obstetricia Et Gynecologica Scandinavica. 2018;97:1168-77.

25. Nadaraja S, Jorgensen TL, Matzen L-E, Herrstedt J. Impact of Age, Comorbidity, and FIGO Stage on Treatment Choice and Mortality in Older Danish Patients with Gynecological Cancer: A Retrospective Register-Based Cohort Study. Drugs - real world outcomes. 2018;5:225-35.

26. van Doorn HC, Opmeer BC, Duk MJ, Kruitwagen R, Dijkhuizen F, Mol BW, Dupomeb. The relation between age, time since menopause, and endometrial cancer in women with postmenopausal bleeding. Int J Gynecol Cancer. 2007;17:1118-23. 
27. Nagle CM, Crosbie EJ, Brand A, Obermair A, Oehler MK, Quinn M, Leung Y, Spurdle AB, Webb PM. Australian Natl Endomet Canc S: The association between diabetes, comorbidities, body mass index and all-cause and cause-specific mortality among women with endometrial cancer. Gynecol Oncol. 2018;150:99-105.

28. Modesitt SC, Geffel DL, Via J, Weltman AL. Morbidly obese women with and without endometrial cancer: Are there differences in measured physical fitness, body composition, or hormones? Gynecol Oncol. 2012;124:431-6.

29. Yin XH, Jia HY, Xue XR, Yang SZ, Wang ZQ. Clinical analysis of endometrial cancer patients with obesity, diabetes, and hypertension. International Journal of Clinical Experimental Medicine. 2014;7:736-43.

30. Nimptsch K, Pischon T. Body fatness, related biomarkers and cancer risk: an epidemiological perspective. Horm Mol Biol Clin Investig. 2015;22:39-51.

31. Papanas N, Giatromanolaki A, Galazios G, Maltezos E, Sivridis E. Endometrial carcinoma and diabetes revisited. Eur J Gynaecol Oncol. 2006;27:505-8.

32. Soisson S, Ganz PA, Gaffney D, Rowe K, Snyder J, Wan Y, Deshmukh V, Newman M, Fraser A, Smith K, et al. Long-term Cardiovascular Outcomes Among Endometrial Cancer Survivors in a Large, Population-Based Cohort Study. J Natl Cancer Inst. 2018;110:1342-51.

33. Schildkraut JM, Thompson WD. Relationship of epithelial ovarian cancer to other malignancies within families. Genet Epidemiol. 1988;5:355-67.

34. Liu C, Xu J, Wen F, Yang F, Li X, Geng D, Li L, Chen J, Zheng J. Upregulation of syncytin-1 promotes invasion and metastasis by activating epithelial-mesenchymal transition-related pathway in endometrial carcinoma. OncoTargets therapy. 2019;12:31-40.

35. Hosseini-Beheshti E, Choi W, Weiswald LB, Kharmate G, Ghaffari M, Roshan-Moniri M, Hassona MD, Chan L, Chin MY, Tai IT, et al. Exosomes confer pro-survival signals to alter the phenotype of prostate cells in their surrounding environment. Oncotarget. 2016;7:14639-58.

36. Liu Z-M, Wang Y-B, Yuan X-H. Exosomes from Murine-derived GL26 Cells Promote Glioblastoma Tumor Growth by Reducing Number and Function of CD8 + T Cells. Asian Pac J Cancer Prev. 2013;14:309-14.

37. Shi J, Ren Y, Zhen L, Qiu X. Exosomes from breast cancer cells stimulate proliferation and inhibit apoptosis of CD133(+) cancer cells in vitro. Mol Med Rep. 2015;11:405-9.

38. Liu Y, Cao X. Organotropic metastasis: role of tumor exosomes. Cell Res. 2016;26:149-50.

39. Guo FJ, Zhang HN, Jia ZH, Cui MH, Tian JY. Chemoresistance and targeting of growth factors/cytokines signalling pathways: towards the development of effective therapeutic strategy for endometrial cancer. American Journal of Cancer Research. 2018;8:1317-31.

40. Felix AS, Cohn DE, Brasky TM, Zaino R, Park K, Mutch DG, Creasman WT, Thaker PH, Walker JL, Moore RG, et al: Receipt of adjuvant endometrial cancer treatment updates according to race: an NRG Oncology/Gynecologic Oncology Group 210 Study. American Journal of Obstetrics and Gynecology 2018, 219. 
41. Van Nyen T, Moiola CP, Colas E, Annibali D, Amant F. Modeling Endometrial Cancer: Past, Present, and Future. International Journal of Molecular Sciences 2018, 19.

42. Hogberg T. Adjuvant chemotherapy in endometrial carcinoma: Overview of randomised trials. Clin Oncol. 2008;20:463-9.

43. Bogliolo S, Cassani C, Gardella B, Musacchi V, Babilonti L, Venturini PL, Ferrero S, Spinillo A. Current opinion on bevacizumab on endometrial cancer treatment. Expert Opinion on Biological Therapy. 2015;15:299-307.

44. Zhou X, Gao Q, Wang JZ, Zhang X, Liu K, Duan Z. Linc-RNA-RoR acts as a "sponge" against mediation of the differentiation of endometrial cancer stem cells by microRNA-145. Gynecol Oncol. 2014;133:333-9.

45. Vallone C, Rigon G, Gulia C, Baffa A, Votino R, Morosetti G, Zaami S, Briganti V, Catania F, Gaffi M, et al: Non-Coding RNAs and Endometrial Cancer. Genes 2018, 9.

\section{Figures}


a

\section{Volcano}

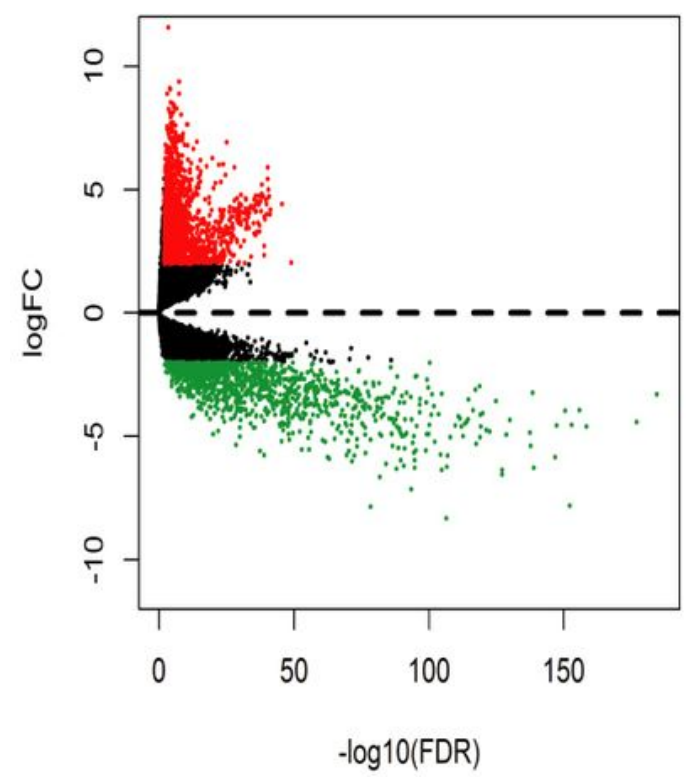

C

Volcano

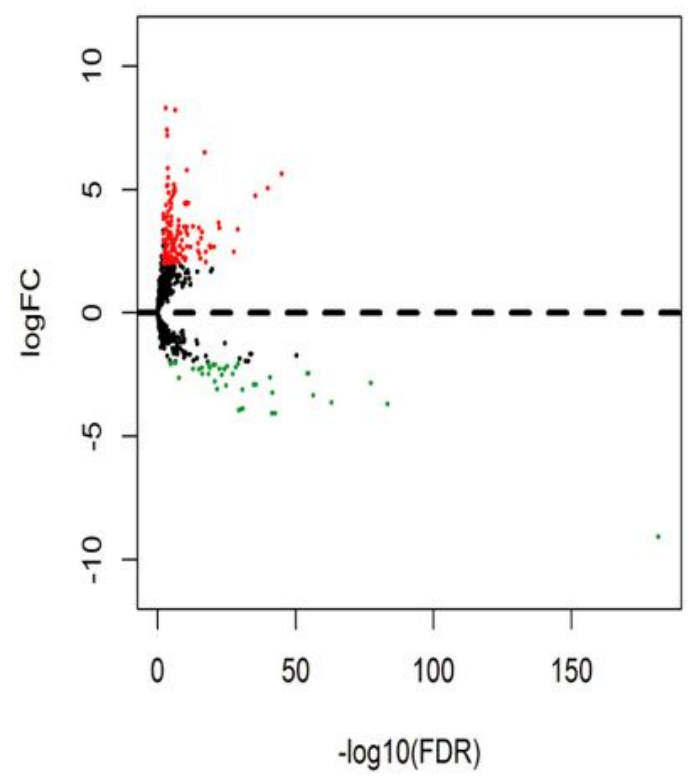

$\mathrm{b}$

\section{Volcano}

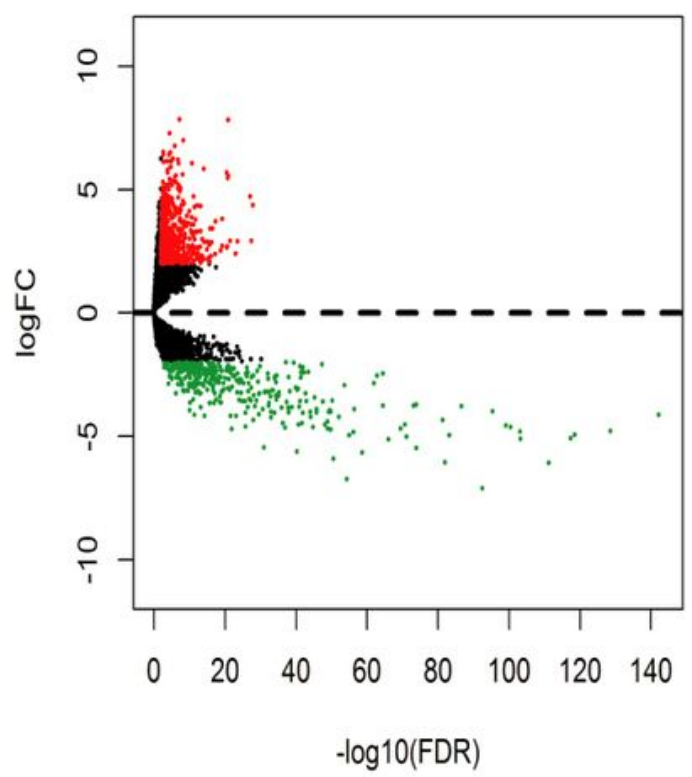

d

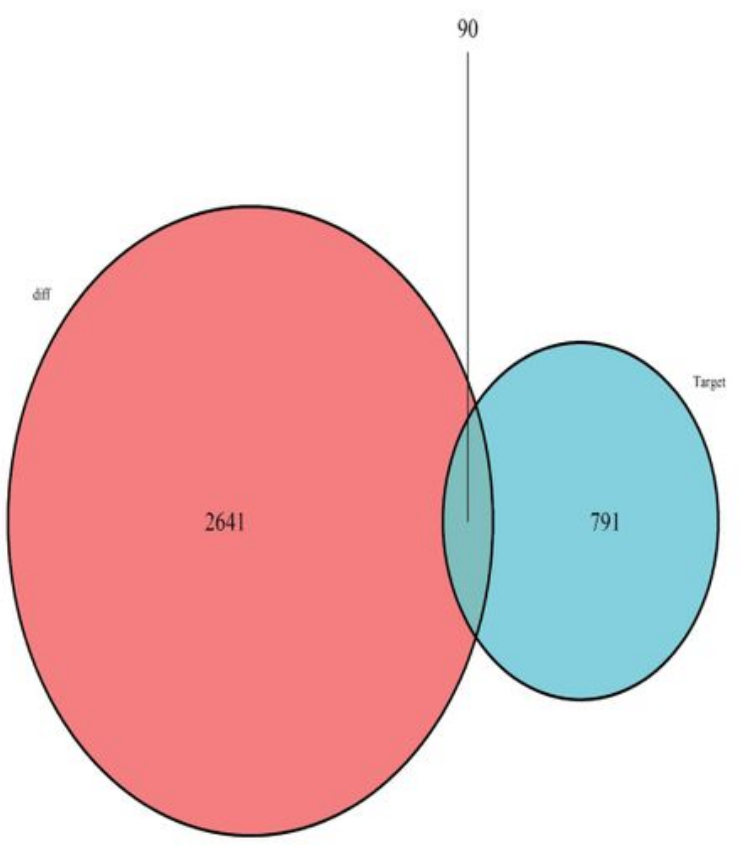

\section{Figure 1}

Volcano plot and Venn diagram of differentially expressed RNAs in endometrial carcinoma (EC). (a) Volcano plot visualizing differentially expressed genes (DEGs) at the mRNA level (19 normal samples and 406 EC samples); the horizontal line marks a - $\log 10$-adjusted P-value of 0.01 , the red spots indicate upregulated expression, and the green spots indicate downregulated expression. (b) Volcano plot visualizing DEGs at the IncRNA level (19 normal samples and 406 EC samples). (c) Volcano plot 
visualizing DEGs at the miRNA level (18 normal samples and 405 EC samples). (d) Venn diagram representing the intersection of predicted target genes and the screened differentially expressed RNAs.

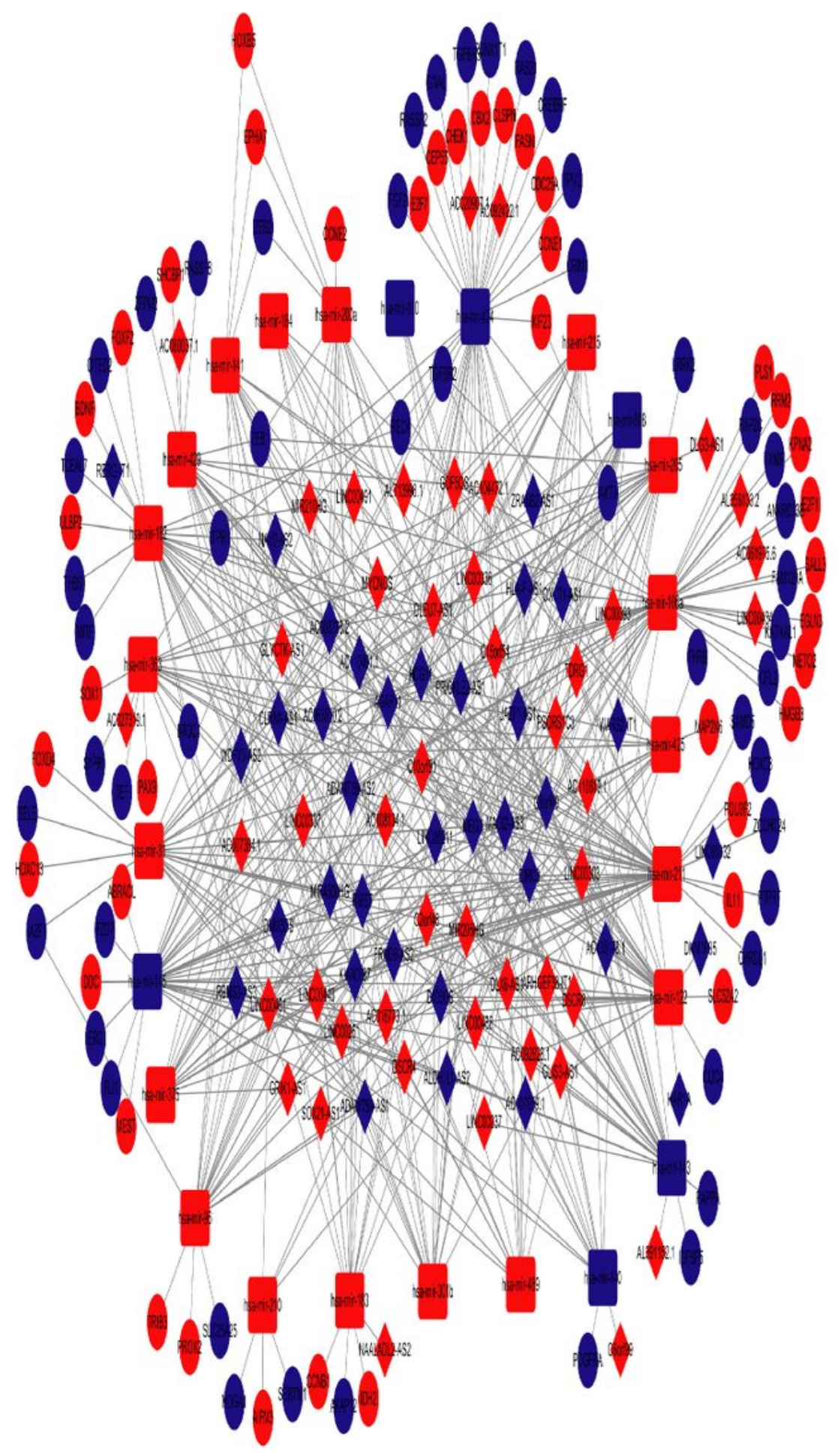

Figure 2

CeRNA network in endometrial carcinoma. The blue nodes represent decreased expression levels, and the red nodes represent increased expression levels. Rectangles represent miRNAs, ellipses represent 
protein-coding genes, and diamonds represent IncRNAs; gray edges indicate IncRNA-miRNA-mRNA interactions.

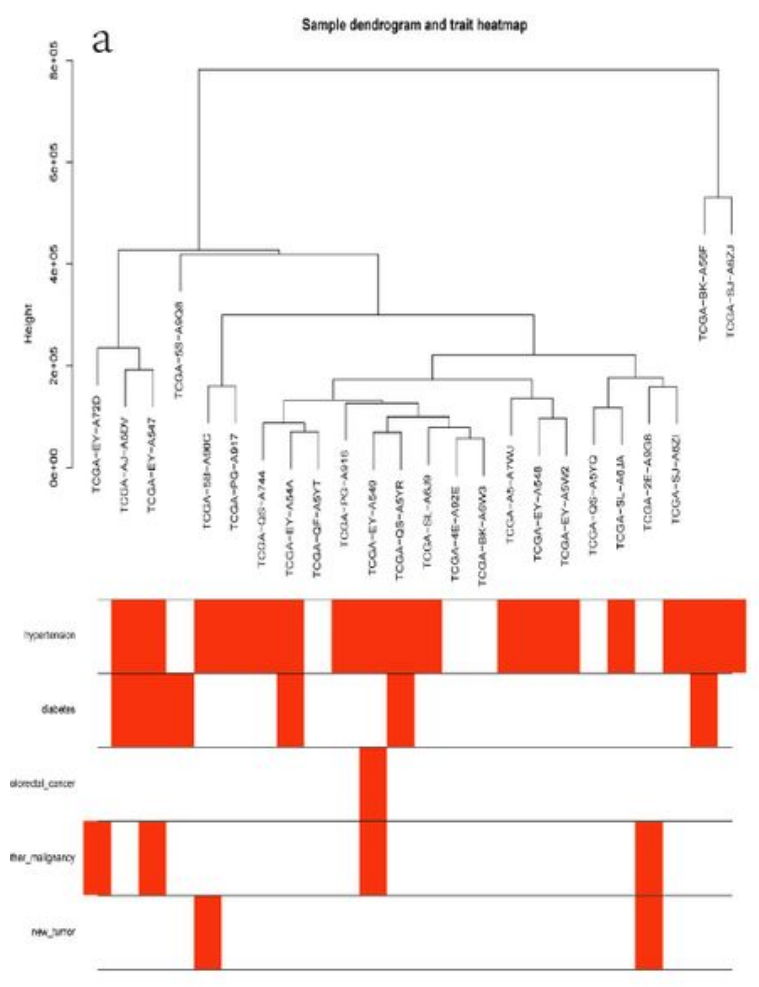

C

Scale independence

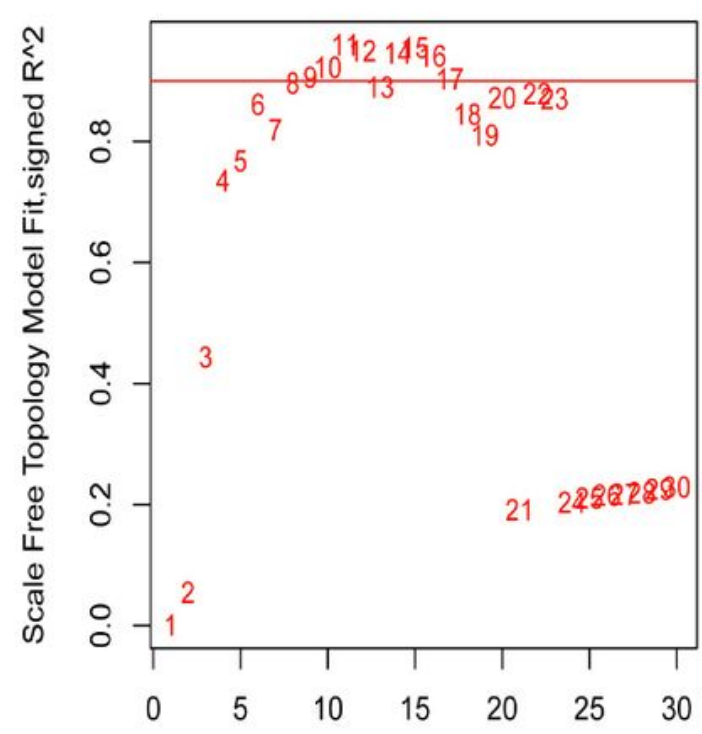

Soft Threshold (power)

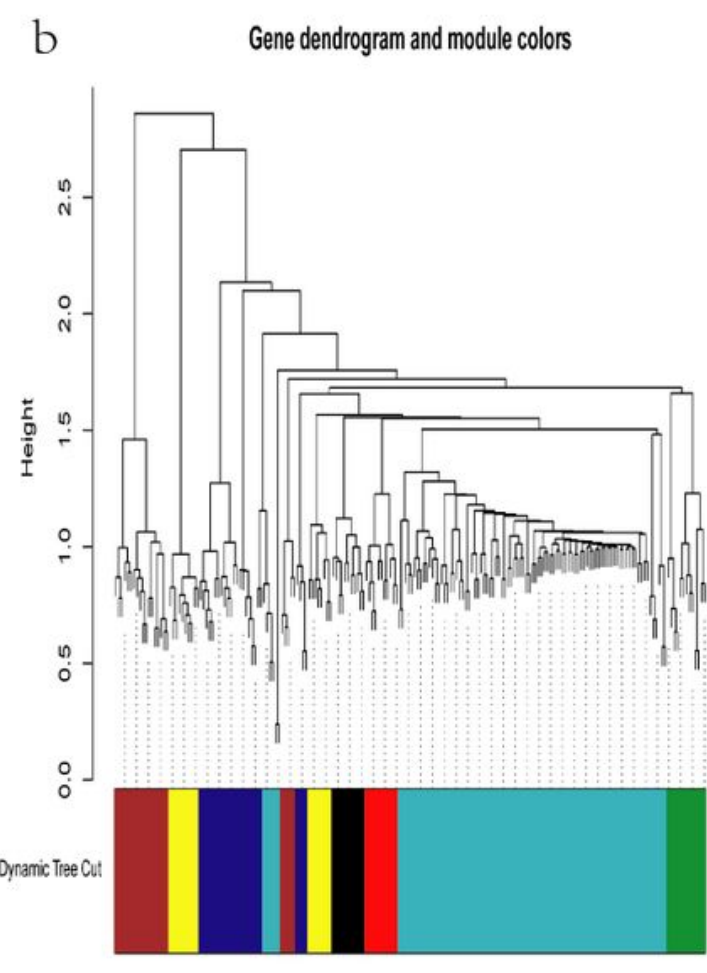

d

Mean connectivity

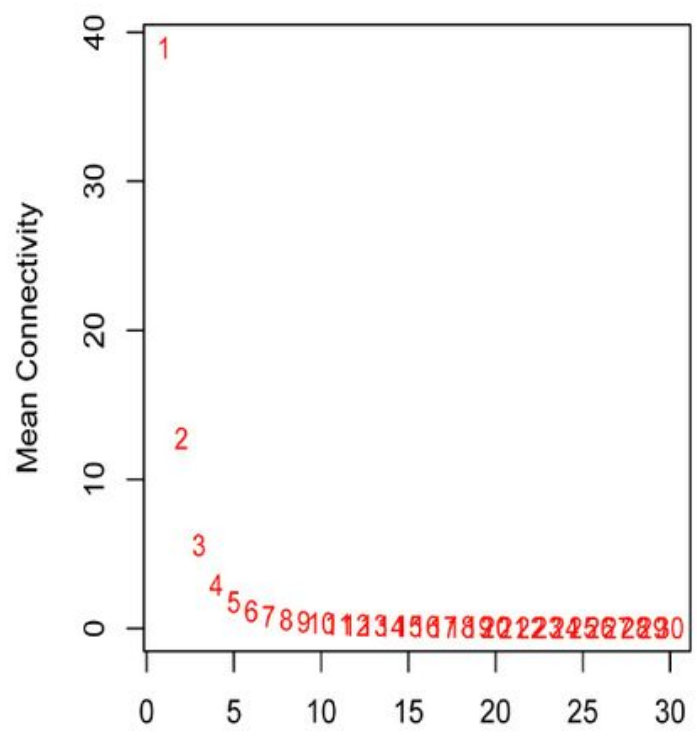

Soft Threshold (power)

\section{Figure 3}

(a) Sample clustering and corresponding heat map of trait data after removing abnormal samples. The color intensity is proportional to hypertension, diabetes, history of colorectal cancer, history of other malignancies, or a new tumor event. (b) Cluster dendrogram of genes in 26 TCGA samples. Each branch 
represents one gene, and every color below represents one co-expression module. (c) Analysis of the scale-free ft index for various soft-thresholding powers $(\beta)$. (d) Analysis of the mean connectivity for various soft-thresholding powers.

a

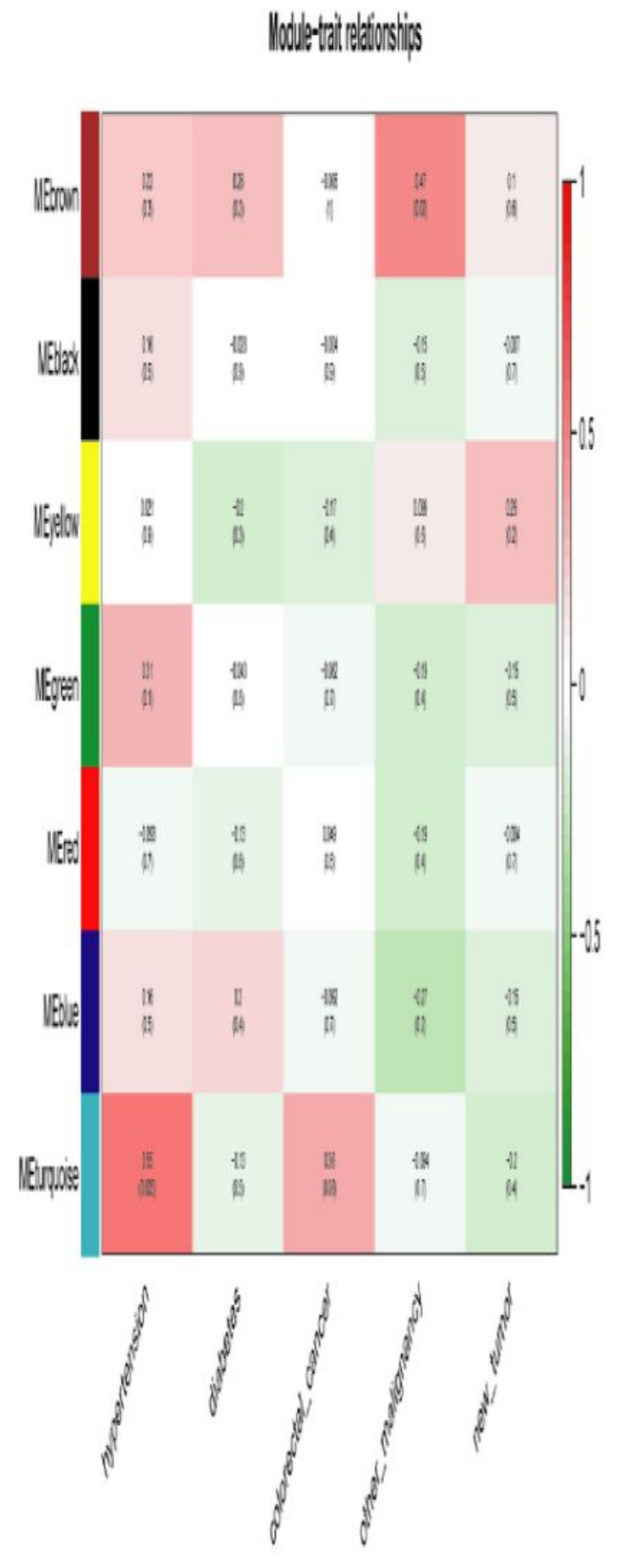

b

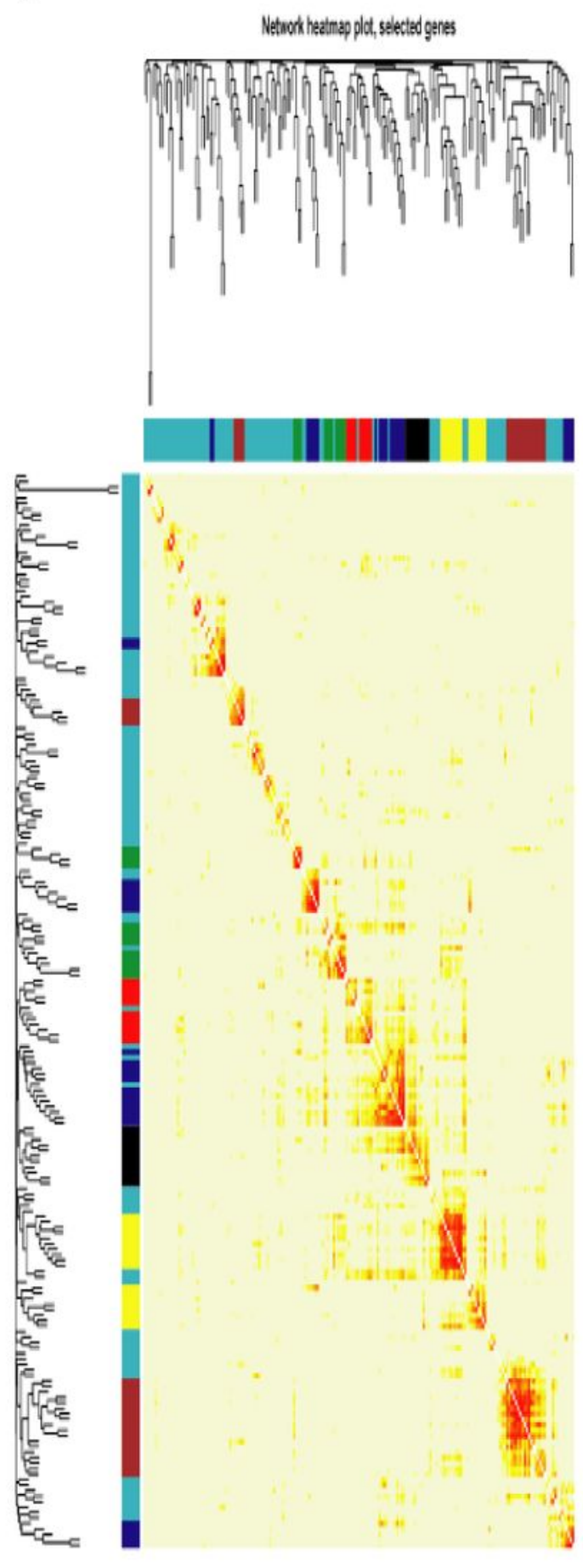

Figure 4

(a) Heatmap of the correlation between module eigengenes and the disease status of endometrial cancer.

(b) Interaction relationship analysis of co-expressed genes. Different colors of the horizontal axis and 
vertical axis represent different modules. The brightness of yellow in the middle represents the degree of connectivity of different modules. There was no significant difference in interactions among different modules, indicating a high-scale independence degree among these modules.
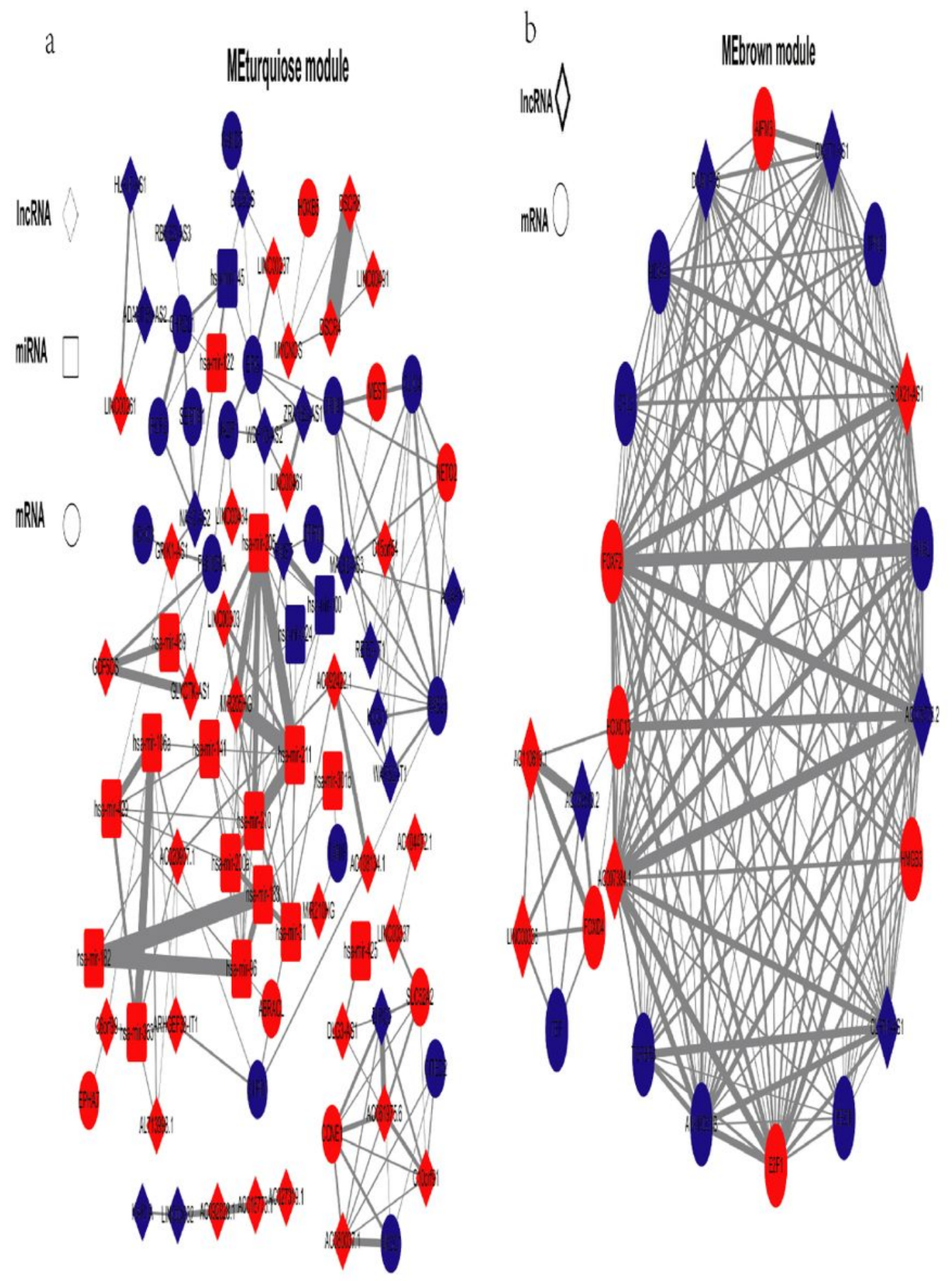

Figure 5

CeRNA network in the MEturquoise and MEbrown modules in endometrial carcinoma. The blue nodes represent decreased expression levels, and the red nodes represent increased expression levels. 
Rectangles represent miRNAs, ellipses represent protein-coding genes, and diamonds represent IncRNAs; gray edges indicate IncRNA-miRNA-mRNA interactions. The edge width is correlated with the degree of connectivity of the gene.

a

GO:2000020 positive regulation of male gonad development

GO:0060412 ventricular septum morphogenesis GO:0060045 positive regulation of cardiac muscle cell proliferation

GO:0045944 positive regulation of transcription from RNA polymerase II promote

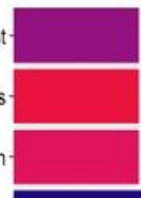

G0:0045446 endothelial cell differentiation

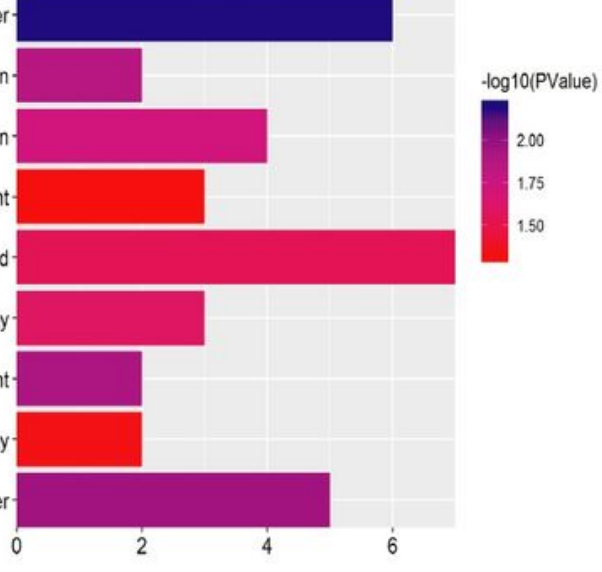

b

G0:0045944 positive regulation of transcription from RNA polymerase II promoter
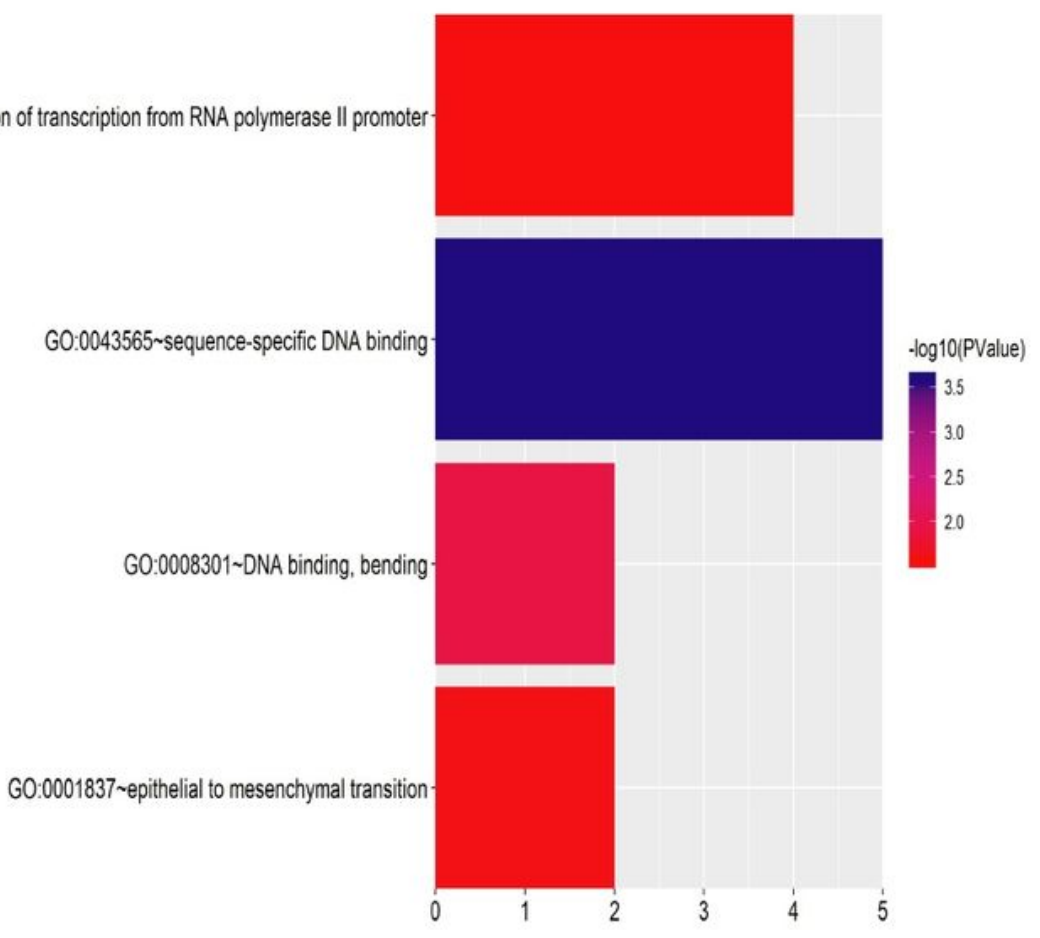

\section{Figure 6}

Significantly enriched GO terms of the MEturquiose and MEbrown modules for differentially expressed ceRNAs in endometrial carcinoma (EC). Gene Ontology (GO) enrichment analysis was performed using 
DAVID. A P-value was obtained using a hypergeometric test; $\mathrm{P}<0.05$ was set as the cut-off for significant $\mathrm{GO}$ terms. The $y$-axis shows $\mathrm{GO}$ terms and the $\mathrm{x}$-axis presents the counts of differentially expressed ceRNAs in EC-enriched GO terms. The color scale represents the -log false discovery rate (FDR). (a) MEturquiose module; (b) MEbrown module.
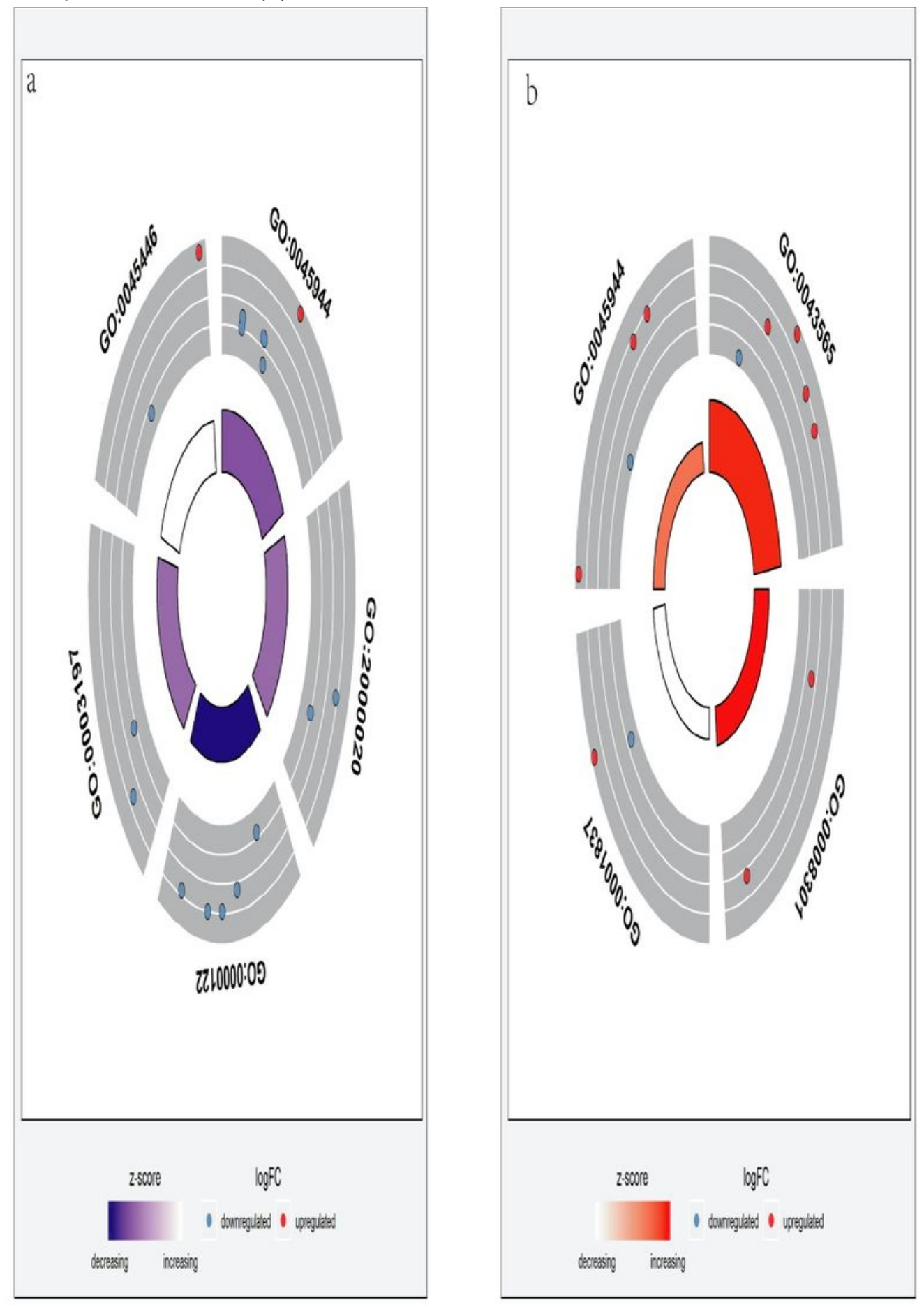

Figure 7 
Gene Ontology (GO) enrichment of genes in the (a) MEturquiose module and (b) MEbrown module. Visualization was performed using the GO-plot package in R. The outer circle shows a scatter plot of the log-fold change for the assigned genes within each distinct GO term. Selection for enrichment resulting in blue and red dots indicates downregulated and upregulation expression, respectively. The inner circle is a bar plot with the height of the bar illustrating the p-value of the term and the color representing the $z$ score, which indicates the direction of the fold change of each of the genes within the assigned term and provides a relative scale for enrichment.


$\mathrm{k}$

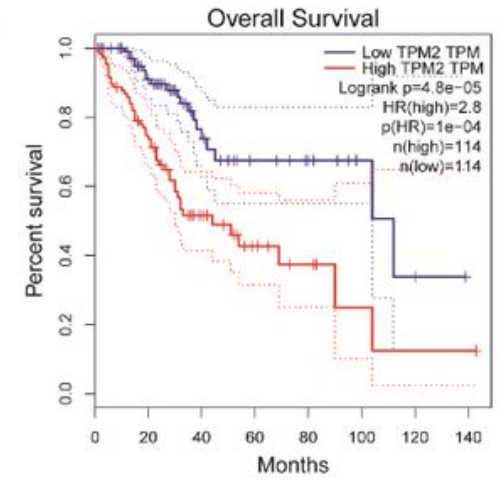


Figure 8

Survival analysis of hub genes. $(a-f)$ Six hub genes in the MEturquoise module with the highest node degree and significant results in the survival analysis $(P<0.05)$ : C10orf91, LINC00303, DIRC3, DLG3-AS1, ARHGEF38-IT1, and CCNE1, respectively. ( $g-k$ ) Five hub genes in the MEbrown module with the highest node degree and significant results in the survival analysis $(P<0.05)$ : RECK, MDGA1, CFL2, TGFBR3, and TPM2, respectively.
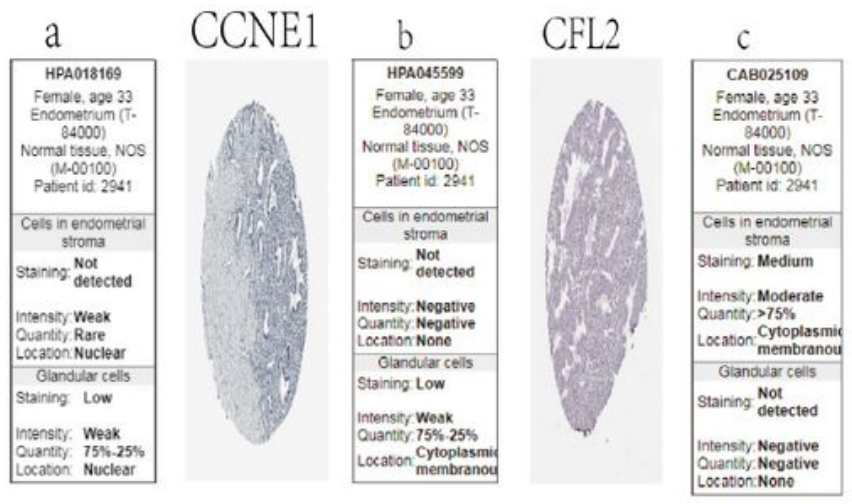

RECK
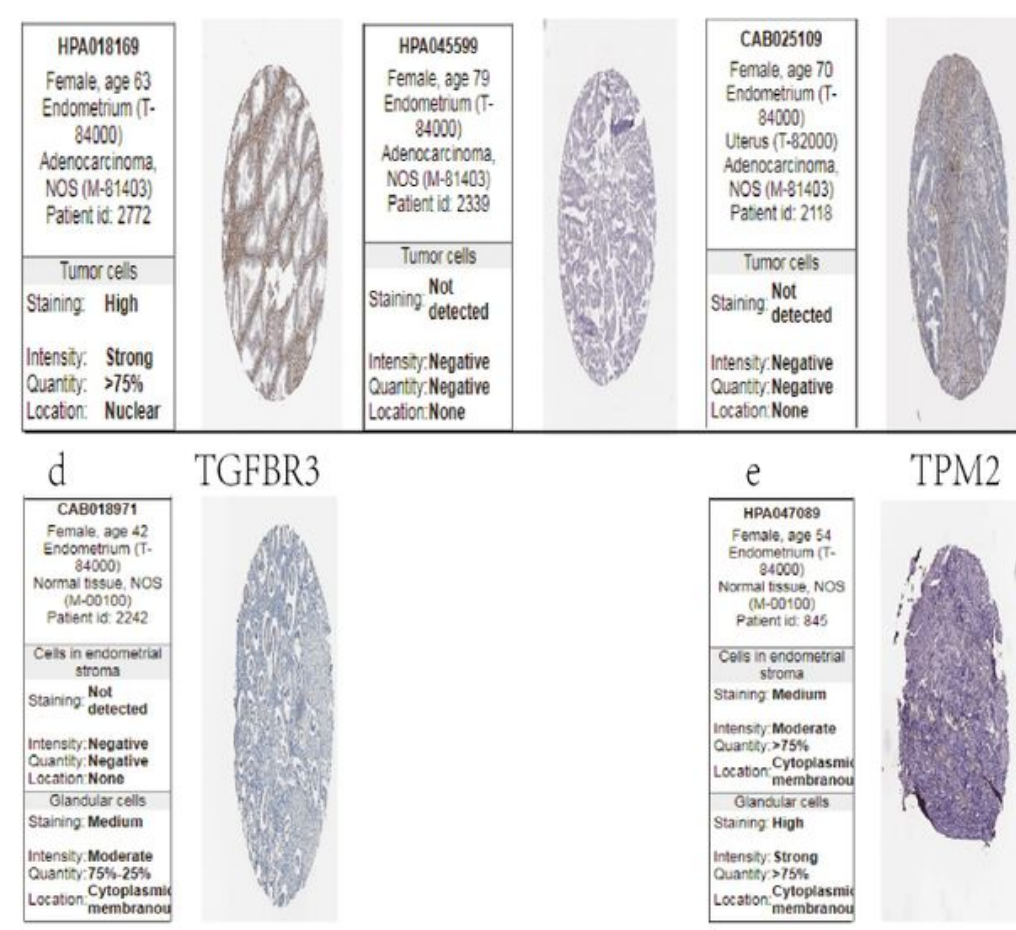

\begin{tabular}{|c|}
\hline CAB018971 \\
Female, age 70 \\
Endometrium (T. \\
84000) \\
Uterus (T-82000) \\
Adenocarcinoma, \\
NoS (M-81403) \\
Patient id: 2118 \\
\hline Tumor cells \\
Staining. Not \\
detected \\
Intensity. Negative \\
Quantlyy. Negative
\end{tabular}
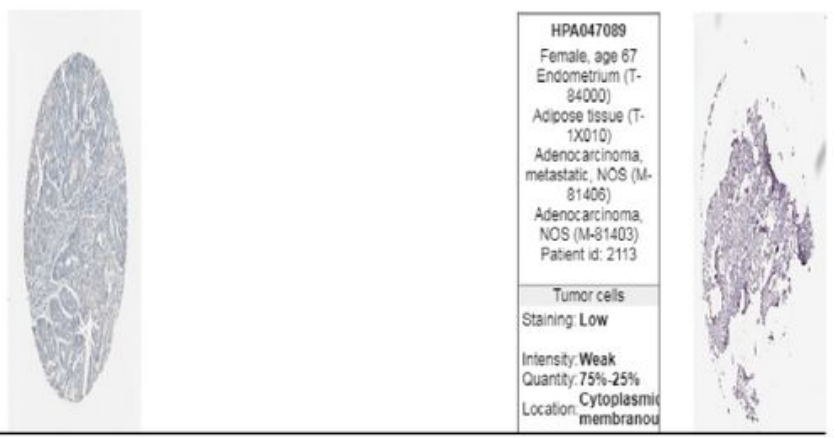


\section{Figure 9}

Validation of hub genes at the translational level. $(a-d)$ Validation of five hub genes in the MEturquoise module by The Human Protein Atlas database (based on immunohistochemistry, IHC). There was no related IHC samples of MMP1 in the database. The translational expression level of the remaining four hub genes was positively correlated with disease status as they were upregulated in oral squamous cell carcinoma (OSCC) samples. (e-i) Validation of five hub genes in the MEbrown module by The Human Protein Atlas database. The translational expression level of five hub genes was negatively correlated with disease status, as they were downregulated in OSCC samples. 
a

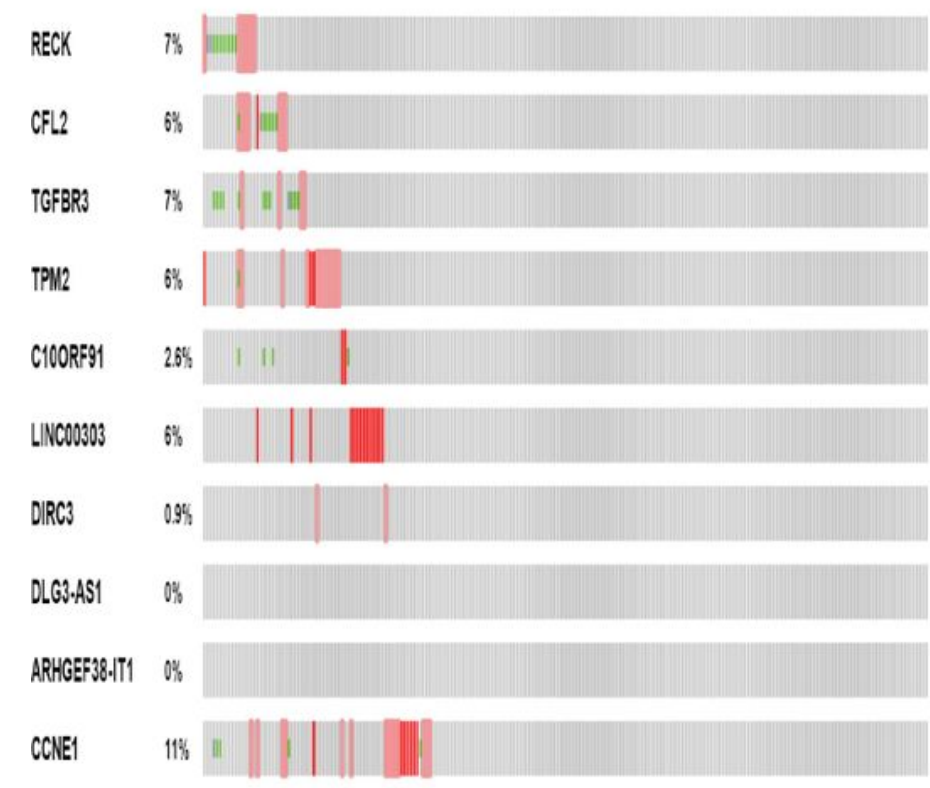

b

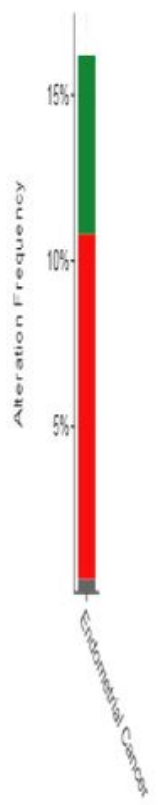

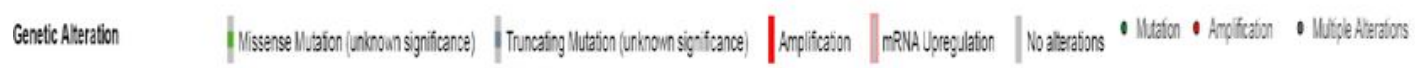

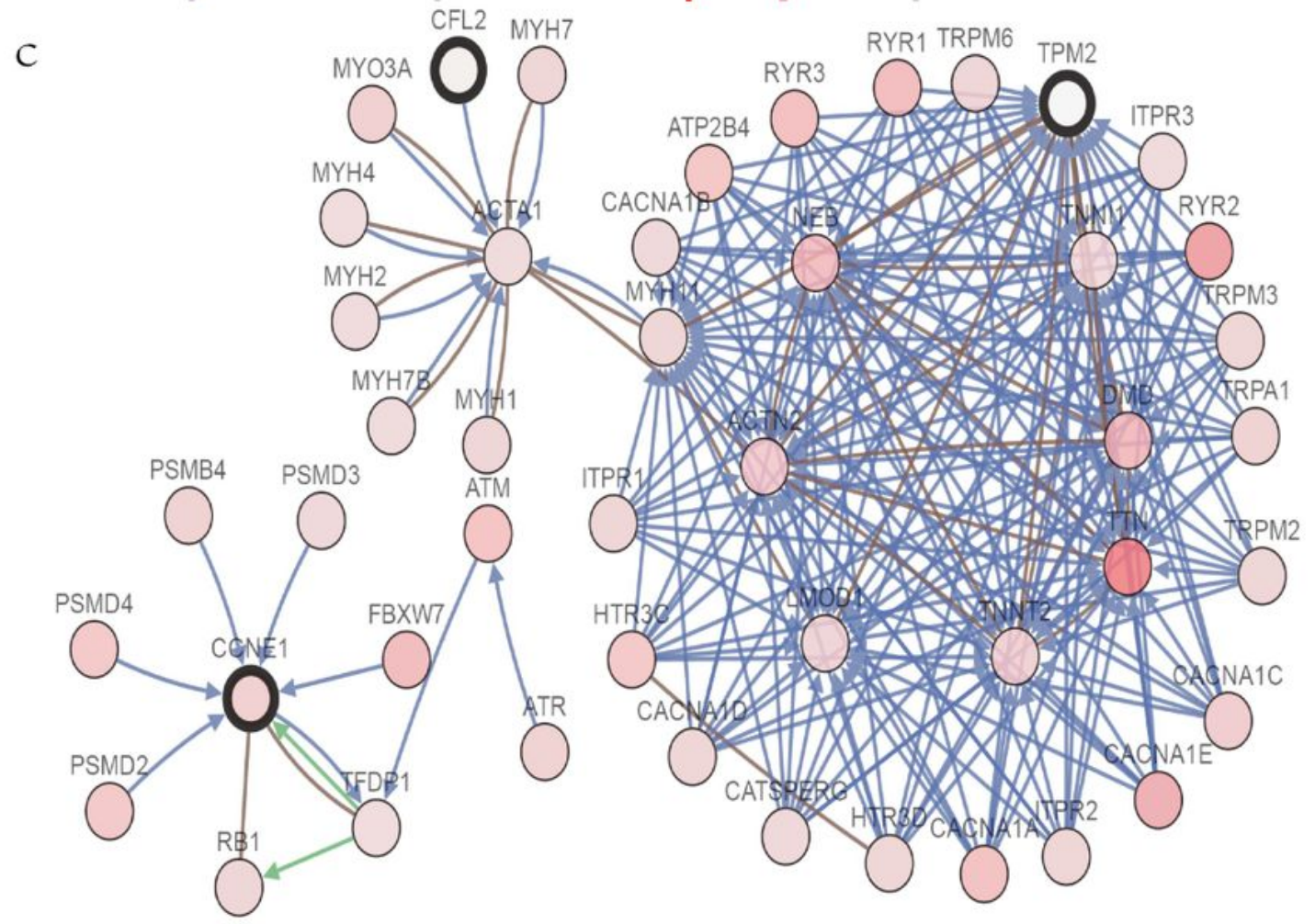

\section{Figure 10}

Genetic alterations associated with hub genes in the TCGA endometrial carcinoma (EC) database. (a) Visual summary of the genetic alterations of 10 hub genes in TCGA EC patients. (b) Total alteration frequency of 10 hub genes in TCGA EC. (C) The network contains 60 nodes, including our 10 query genes and the 50 most frequently altered neighbor genes (only three out of the 10 hub genes were correlated with the 50 genes). 

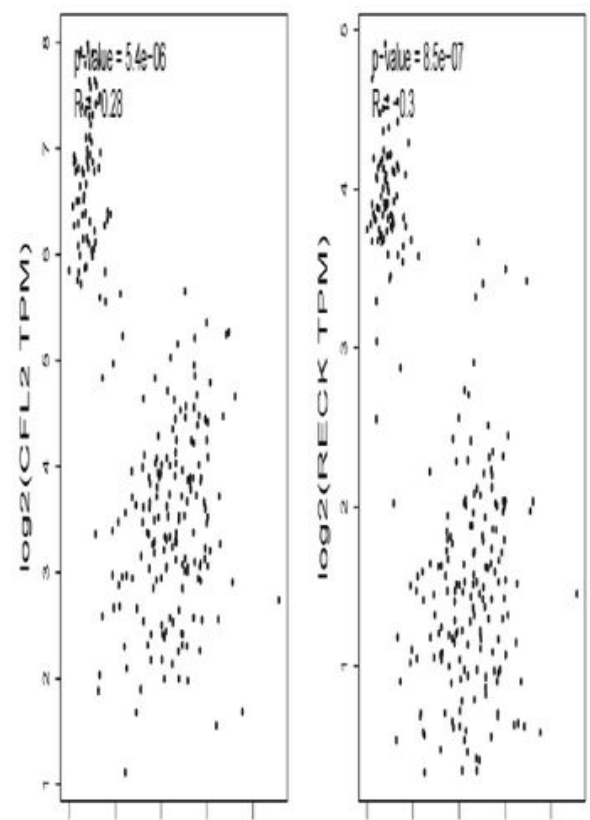

WOACAET:TPII

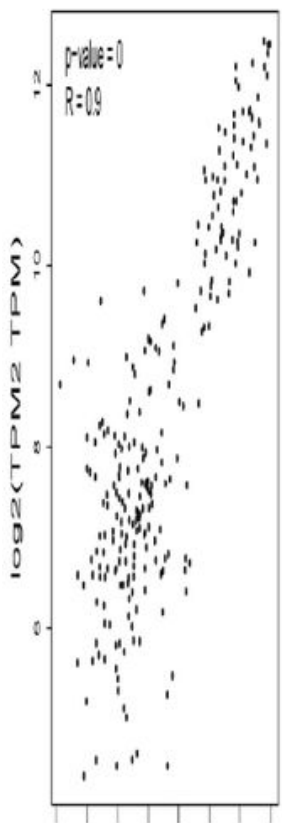

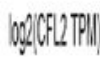

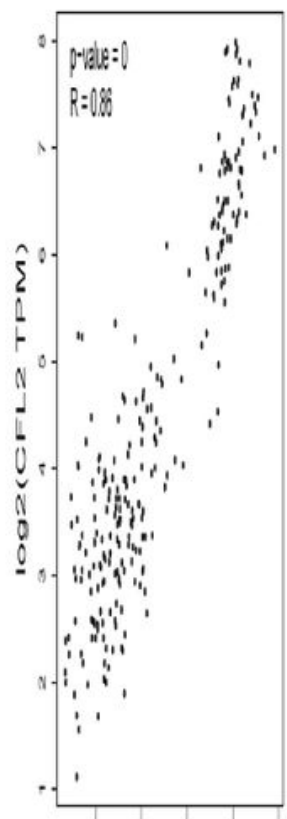

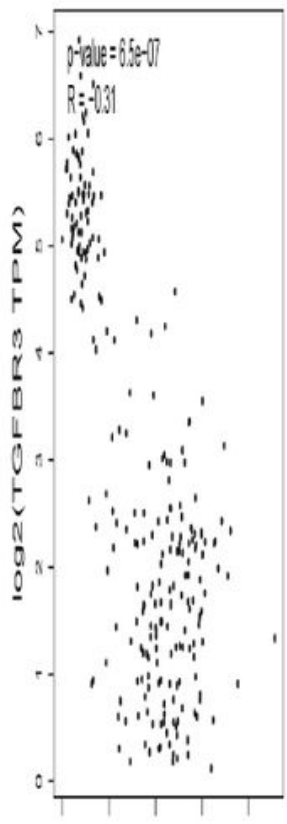
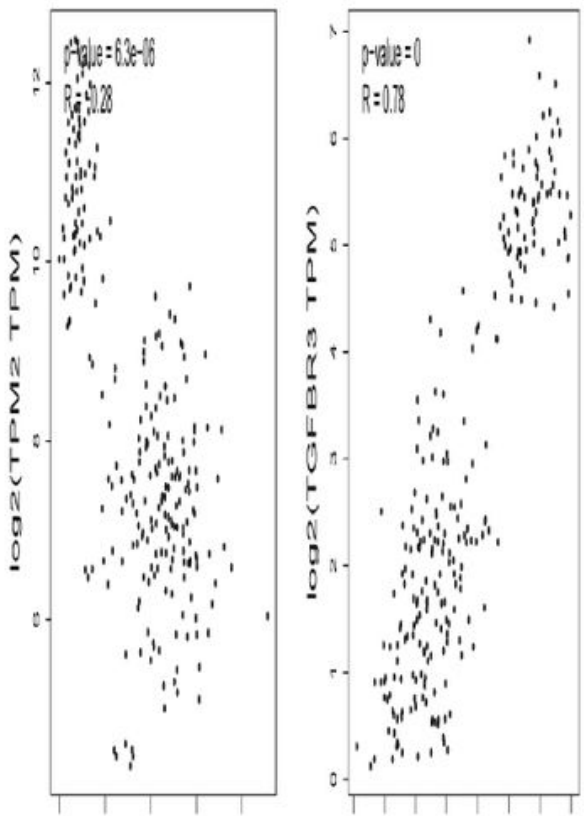

ogeCCNET TPYII)
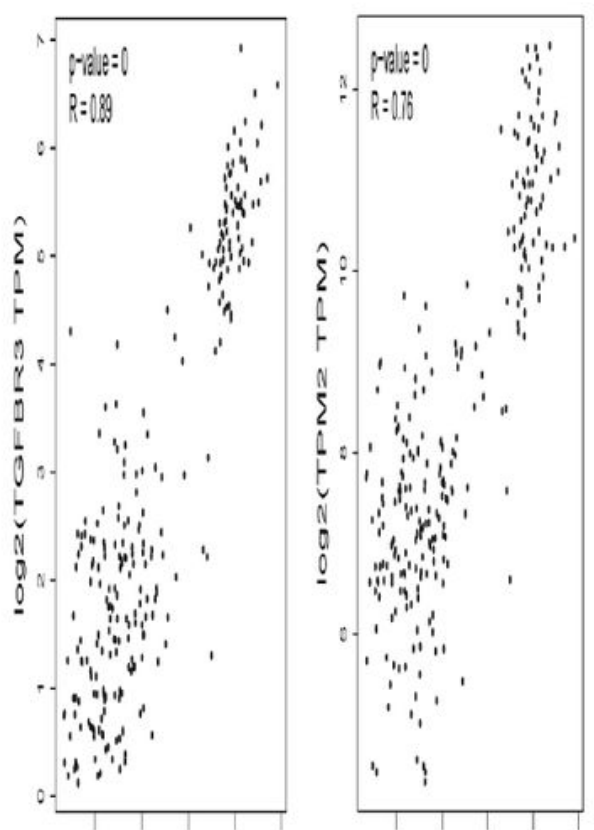

WOQRECX TPMI

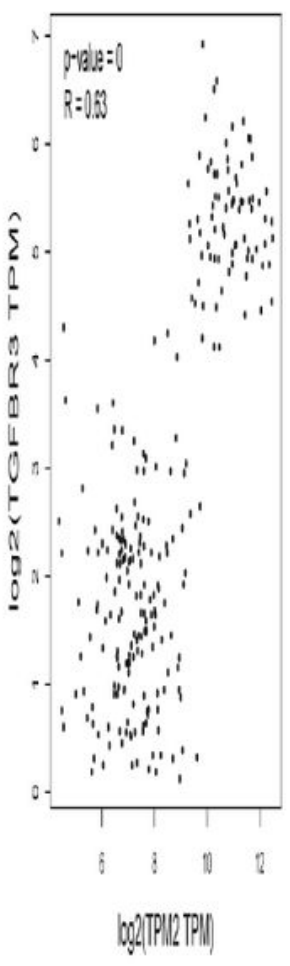

\section{oge(CELTPU)}

\section{Figure 11}

The correlation analysis of the mRNAs RECK, CFL2, TGFBR3, TPM2, and CCNE1 in the GEPIA website.

\section{Supplementary Files}

This is a list of supplementary files associated with this preprint. Click to download. 
- 13.jpg

- 12.txt

- 11.txt

- 10.xls

- 9.xls

- 8.xls

- 7.xls

- 6.xls

- 5.xls

- 4.xls

- 3.xls

- 2.xls

- 1.xls

- TitlesofAdditionalMaterials.docx 